Rodrigo Silva Mello

\title{
Codificação Distribuída de Vídeo com Modelagem do Canal de Correlação no Domínio da Transformada
}

Tese apresentada ao Programa de Pós-graduação em Engenharia Elétrica da PUC-Rio como requisito parcial para obtenção do título de Doutor em Engenharia Elétrica.

Orientador: Marco Antonio Grivet Mattoso 


$$
\text { Pontifícia } U_{\text {Niversidade }} \text { Católica }_{\text {Do Rio de Janeiro }}
$$

\section{Rodrigo Silva Mello}

\section{Codificação Distribuída de Vídeo com Modelagem do Canal de Correlação no Domínio da Transformada}

Tese apresentada como requisito parcial para obtenção do grau de Doutor pelo Programa de Pós-Graduação em Engenharia Elétrica do Departamento de Engenharia Elétrica do Centro Técnico Científico da PUC-Rio. Aprovada pela Comissão Examinadora abaixo assinada.

Prof. Marco Antonio Grivet Mattoso Maia

Orientador

Centro de Estudos em Telecomunicações /PUC-Rio

Profa Carla Liberal Pagliari

IME

Prof. Lisandro Lovisolo

UERJ

Prof. Marcelo Roberto P. Baptista Jimenez Centro de Estudos em Telecomunicações /PUC-Rio

Prof. Weiler Alves Finamore Centro de Estudos em Telecomunicações /PUC-Rio

Prof. Rodolfo Sabóia Lima de Souza Inmetro

Prof. José Eugenio Leal Coordenador Setorial do Centro

Técnico Científico - PUC-Rio

Rio de Janeiro, 16 de dezembro de 2010 
Todos os direitos reservados. É proibida a reprodução total ou parcial do trabalho sem autorização do autor, do orientador e da universidade.

\section{Rodrigo Silva Mello}

possui curso técnico em Mecânica Industrial pelo Centro Federal de Educação Tecnológica Celso Suckow da Fonseca (1997), graduação em Engenharia Elétrica com ênfase em Telecomunicações pela Universidade do Estado do Rio de Janeiro (2002), mestrado em Engenharia Elétrica (Telecomunicações) pela Pontifícia Universidade Católica do Rio de Janeiro (2005) e doutorado em Engenharia Elétrica (Telecomunicações) pela Pontifícia Universidade Católica do Rio de Janeiro (2010). Tem experiência na área de Engenharia Elétrica, com ênfase em Sistemas de Telecomunicações e Processamento de Sinais, atuando principalmente nos seguintes temas: Codificação de Vídeo Digital, Sistemas Móveis Celulares e Rádio Troncalizado, Telefonia IP e Comunicação de Dados pela Rede Elétrica (PLC).

Ficha Catalográfica

Mello, Rodrigo Silva

Codificação distribuída de vídeo com modelagem do canal de correlação no domínio da transformada / Rodrigo Silva Mello; orientador: Marco Antonio Grivet Mattoso. - 2010.

209 f. il. (color.); $30 \mathrm{~cm}$

Tese (doutorado)-Pontifícia Universidade Católica do Rio de Janeiro, Departamento de Engenharia Elétrica, 2010.

Inclui bibliografia

1. Engenharia elétrica - Teses. 2. Codificação distribuída de vídeo. 3. Teorema de Slepian-Wolf. 4. Teorema de Wyner-Ziv. 5. Informação lateral. 6. Quadro Wyner-Ziv. 7. Código de canal. 8. Plano de bits. 9. Canal de correlação. 10. Interpolação de quadros. I. Mattoso, Marco Antonio Grivet. II. Pontifícia Universidade Católica do Rio de Janeiro. Departamento de Engenharia Elétrica. III. Título. 


\section{Agradecimentos}

A Deus e à Nossa Senhora, pelas bênçãos e graças que me sustentaram em todos os momentos, ajudando-me a superar obstáculos para a realização deste estudo.

Aos meus pais Fernando e Bernardete, pelo amor, carinho educação, empenho e pelo sustento proporcionados ao longo dessa difícil jornada.

Aos meus irmãos, Fernanda e Ramon e demais familiares, pelo apoio, compreensão e incentivo durante todo este tempo.

Ao meu orientador, Professor Marco Antonio Grivet Mattoso, pela sua competência e por seu papel fundamental na estruturação deste trabalho, com sua orientação e dedicação incansáveis.

Aos professores Carla Liberal Pagliari e Lisandro Lovisolo, pela cooperação nos estudos desenvolvidos.

À professora Ana Pavani, pela amizade e grande parceria no encaminhamento das questões para alcance dos objetivos.

À PUC-Rio, e em especial ao corpo docente do CETUC, pela contribuição dada ao meu aprendizado profissional na Área de Telecomunicações.

Aos colegas do CETUC pelo apoio em todos os momentos.

Aos meus amigos em geral, pela amizade e estímulo constantes. 


\section{Resumo}

Mello, Rodrigo Silva; Mattoso, Marco Antonio Grivet. Codificação distribuída de vídeo com modelagem do canal de correlação no domínio da transformada. Rio de Janeiro, 2010. 209p. Tese de Doutorado Departamento de Engenharia Elétrica, Pontifícia Universidade Católica do Rio de Janeiro.

A codificação distribuída de vídeo é um novo paradigma de codificação de vídeo que permite explorar a estatística da fonte apenas no decodificador. Nesse cenário, duas fontes correlatadas são independentemente codificadas usando codificadores distintos e os fluxos binários associados a cada uma são conjuntamente decodificados, explorando a correlação entre eles. A resiliência a erros é uma importante funcionalidade deste novo paradigma uma vez que a tradicional malha de predição no codificador e a propagação de erros associada a esta malha não existe ao não se explorar a correlação do sinal no codificador. Desta forma, esta tese tem como objetivo apresentar uma nova arquitetura de codificação distribuída de vídeo, desenvolvendo e analisando algoritmos mais eficientes para a mesma, reduzindo, desta forma, o gap de desempenho quando comparado aos sistemas tradicionais de codificação de vídeo [68]. Assim, o codec proposto nesta tese proporcionou: a otimização da arquitetura DVC (Distributed Vídeo Coding), o desenvolvimento de novas ferramentas para a geração da informação lateral, a inclusão de um módulo de medida de correlação entre quadros para auxiliar na decodificação, a utilização de um processo de quantização Wyner-Ziv variável, a opção de uma codificação parcial de bitplanes, uma modelagem do canal de correlação baseada na estimação de parâmetros em nível de banda e de coeficientes da transformada DCT (Discrete Cosine Transform), a inclusão de um módulo de normalização da informação lateral e o desenvolvimento de um código de canal mais adequado à arquitetura proposta.

\section{Palavras-chave}

Codificação Distribuída de Vídeo; Teorema de Slepian-Wolf; Teorema de Wyner-Ziv; Informação Lateral; Quadro Wyner-Ziv; Código de Canal; Plano de bits; Canal de Correlação; Interpolação de Quadros. 


\section{Abstract}

Mello, Rodrigo Silva; Mattoso, Marco Antonio Grivet (Advisor). Distributed video coding with correlation channel modeling in the transform domain. Rio de Janeiro, 2010. 209p. Dsc. Thesis Departamento de Engenharia Elétrica, Pontifícia Universidade Católica do Rio de Janeiro.

Distributed video coding is a new video coding paradigm that allows exploiting the source statistics at the decoder only. In this scenario, two correlated sources are independently encoded using separated encoders and the bit streams associated to each one are jointly decoded exploiting the correlation between them. Improved error resilience is another major functionality of this new video coding paradigm since the usual encoder prediction loop and the associated error propagation do not exist anymore, because the signal correlation is not explored in the encoder. Therefore, this thesis aims to present a new architecture for distributed video coding, analyzing and developing more efficient algorithms for it, thus reducing the gap in performance when it is compared to traditional video coding [68]. Therefore, the codec proposed in this thesis provided: a DVC architecture optimization; the development of new tools for side information generation process; inclusion of a module for measuring correlation between frames in order to assist in the Wyner-Ziv frame reconstruction; the use of a Wyner-Ziv variable quantization process; the option of partial coding of bitplanes; a correlation channel model based on the estimation of parameters in band and DCT coefficient levels; an inclusion of a normalization stage to the side information; and the development of a channel code more appropriate to the proposed architecture.

\section{Keywords}

Distributed Video Coding; Slepian-Wolf Theorem; Wyner-Ziv Theorem; Side Information; Wyner-Ziv frame; Channel Code; Bitplanes; Correlation Channel; frame interpolation. 


\section{Sumário}

1 Introdução

1.1. Fundamentos da Codificação Distribuída 26

1.2. Teorema de Slepian-Wolf para Codificação Distribuída 28

1.3. Codificação de Síndrome 30

1.4. Principais Objetivos da Tese 33

1.5. Apresentação do Trabalho 36

$\begin{array}{ll}\text { 1.6. Publicações } & 37\end{array}$

2 Aplicação da Codificação Wyner-Ziv para Vídeo 38

2.1. Cálculo de Taxa-Distorção para Codificação com Informação Lateral no Receptor 38

2.2. Revisão de literatura sobre codificação Wyner-Ziv 40

2.3. Funcionamento da Codificação de Vídeo de Baixa Complexidade 41

2.4. Arquitetura-padrão para Codec DVC 43

3 Código de Baixa Densidade, Irregular e com Taxa Variável 49

3.1. Escolha do código 49

3.2. DVC baseado em Síndromes 49

3.3. Escolha do código 51

3.3.1. Fundamentos da Codificação LDPC Irregular 52

3.3.2. Probabilidades e LLR a posteriori 53

3.3.3. Transferência de Probabilidades entre Nós dos Grafos 54

3.3.4. Regras de Atualização de Mensagens Probabilísticas 57

3.3.5. Método para Síntese de nós de Síndrome 60

3.3.6. Algoritmo de Produto-e-Máximo 62

3.3.6.1. Codificação com o Código LIA 63

3.3.6.2. Decodificação com o Código LIA 65

3.4. Considerações sobre o Código Proposto 68

3.4.1.1. Avaliação da influência das Iterações do Decodificador

LIA na Reconstrução dos Bitplanes 68 
4.1. Motivação 75

4.2. Arquitetura do Codec WZ-LIA 76

4.3. Procedimento de Codificação 77

4.4. Procedimento de Decodificação 80

4.5. Métodos e Técnicas do Codec Proposto 83

4.5.1. Transformada DCT no Codec WZ-LIA 83

4.5.2. Quantizador do Codec WZ-LIA 87

4.5.2.1. Quantização dos Coeficientes DC 87

4.5.2.2. Quantização do Coeficiente AC 91

4.5.3. Número de Níveis de Quantização 97

4.5.4. Abordagem sobre a Faixa Dinâmica 98

4.5.5. Cálculo do Passo de Quantização do Coeficiente DC 100

4.5.6. Cálculo do Passo de Quantização dos Coeficientes AC 102

4.5.7. Descrição do Método Proposto para Quantização 104

4.5.8. Avaliação do Método de Quantização Proposto 107

4.5.9. Procedimento de Geração dos Bitplanes 114

4.5.9.1. Avaliação Objetiva da Transmissão Parcial dos Bitplanes 114

4.5.9.2. Avaliação Subjetiva da Transmissão Parcial dos Bitplanes 117

4.5.10. Procedimento de Geração das Síndromes 118

4.6. Técnicas Propostas para Decodificação 119

4.6.1. Decodificação Iterativa LIA 119

4.6.2. Buffer e Transmissão Parcial das Síndromes 120

4.6.3. Estimação de Taxa Mínima 121

4.6.3.1. Medida de Confiança 125

4.6.4. Modelagem do Canal de Correlação 126

4.6.4.1. Estimação do Ruído de Correlação em nível de

$\begin{array}{ll}\text { Sequência (offline) } & 128\end{array}$

4.6.5. Modelos de Ruído de Correlação Estimados Online 129

4.6.5.1. Estimação do Ruído de Correlação em nível de Frame 129

4.6.5.2. Estimação Proposta para o Ruído de Correlação:

em Nível de Banda 
4.6.5.3. Estimação do Ruído de Correlação em Nível de Bloco

4.6.5.4. Estimação Proposta para o Ruído de Correlação:

$\begin{array}{ll}\text { em Nível de Coeficiente } & 133\end{array}$

4.6.6. Avaliação da Modelagem do canal de Correlação 134

4.6.7. Geração da Informação Lateral 139

4.6.8. Método Proposto para Geração da Informação Lateral 143

4.6.8.1. Estimação Direta de Movimento 143

4.6.8.2. Estimação Reversa do Movimento 148

4.6.9. Avaliação dos Métodos de Estimação Bidirecional $\begin{array}{ll}\text { de Movimento } & 149\end{array}$

4.6.9.1. Problemas da Compensação de Frames Interpolados 159

4.6.9.2. Interpolação com base na Correlação entre Frames 161

4.6.9.3. Interpolação baseada na Estimativa de movimento 162

4.6.9.4. Extrapolação 163

4.6.10. Avaliação Subjetiva do Módulo Proposto para Geração da $\begin{array}{ll}\text { Informação Lateral } & 164\end{array}$

4.7. Avaliação Objetiva das Ferramentas Propostas para o Módulo de Geração da Informação Lateral 167

4.7.1. Avaliação Objetiva do módulo de Normalização 173

4.7.2. Avaliação Subjetiva Conjunta do processo de Normalização e de geração da Informação Lateral 177

$\begin{array}{ll}\text { 4.7.3. Verificador de Falha } & 178\end{array}$

4.7.4. Reconstrução dos Coeficientes DCT 179

4.7.5. Simulações Gerais com o Codec WZ-LIA 183

4.7.5.1. PSNR média em função do Tempo de Processamento 183

4.7.5.2. PSNR ao longo da sequência para Diferentes Taxas de $\begin{array}{ll}\text { Compressão } & 184\end{array}$

4.7.5.3. Avaliação da influência da substituição das bandas zeradas do Frame WZ pela informação Lateral 185

5 Conclusões e Trabalhos Futuros 187

$\begin{array}{ll}\text { 5.1. Sugestões para Trabalhos Futuros } & 190\end{array}$

6 Referências 193 
7 Apêndice A - Construção do Código LDPC

8 Apêndice B - Opções de Configuração do Codec Proposto

9 Apêndice C - Algoritmo Proposto para Decodificação dos Bitplanes 


\section{Lista de figuras}

Figura 1.1: Codificação distribuída de duas sequências aleatórias estatisticamente dependentes, $X$ e $Y$.

Figura 1.2: Teorema de Slepian-Wolf: Região de taxa admissível para codificação distribuída de duas fontes estatisticamente dependentes e igualmente distribuídas.

Figura 1.3: Codificação de uma sequência de símbolos aleatórios X utilizando na decodificação a informação lateral Y correlacionada a X.

Figura 1.4: (a) Codificador e decodificador utilizam a informação lateral $Y$, a qual é correlatada a $X$. Aqui, $X$ pode ser codificado com 2 bits.

(b) Aqui, somente o decodificador tem acesso a $Y$ e ainda $\operatorname{assim} X$ pode ser codificado usando apenas 2 bits.

Figura 2.1: Compressão com perdas de uma sequência X utilizando a informação lateral Y (relacionada estatisticamente a X) no decodificador.

Figura 2.2: Um codificador Wyner-Ziv prático é obtido cascateando um quantizador e um codificador Slepian-Wolf.

Figura 2.3: Arquitetura de transcodificação de vídeo para transmissão wireless, utilizada na rede móvel celular [68].

Figura 2.4: Codificador de vídeo de baixa complexidade e decodificador correspondente.

Figura 2.5: Frames da sequência Salesman, QCIF: (a) Informação lateral $\hat{S}$ no decodificador, gerada por interpolação compensada do movimento (b) Frame reconstruído $S$ ’ depois da decodificação conjunta Wyner-Ziv.

Figura 2.6: Desempenho taxa-distorção de um codec de vídeo

Wyner-Ziv, comparado à codificação de vídeo intraframe

e interframe convencional, para sequência Salesman.

Figura 2.7: Desempenho taxa-distorção de um codec de vídeo

Wyner-Ziv, comparado à codificação de vídeo intraframe

e interframe convencional, para sequência Hall Monitor.

Figura 3.1: Cenário resumido de codificação assimétrica da fonte.

Figura 3.2: Início da difusão de mensagens dos nós de variáveis para 
os nós de paridade.

Figura 3.3: Exemplo de transferência de mensagens entre os nós de grafos bipartidos.

Figura 3.4: As funções VAR e PAR em nós de grau 3. 60

Figura 3.5: Síntese de nó de paridade de grau 3. 62

Figura 3.6: Codificador de baixa densidade, irregular e acumulado. 64

Figura 3.7: Grafo de decodificação das variáveis com o codificador transmitindo todas as síndromes acumuladas.

Figura 3.8: Grafo de codificação com o codificador transmitindo somente as síndromes acumuladas de índice par.

Figura 3.9: Grafo de decodificação com o codificador transmitindo os bits das síndromes de índice par.

Figura 3.10: PSNR do frame Wyner-Ziv para diferentes números de iterações do decodificador LIA, para sequência Foreman, com $\mathrm{QP}=25$ (intra) e $\mathrm{TQ}=18$ (WZ).

Figura 3.11: Diferença entre a PSNR do frame Wyner-Ziv utilizando uma e cinquenta iterações, para a sequência Foreman, com QP = 25 (intra) $\mathrm{e} \mathrm{TQ}=18(\mathrm{WZ})$.

Figura 3.12: PSNR do frame Wyner-Ziv para números diferentes de iterações do decodificador LIA, para sequência News, com QP = 25 (intra) e TQ = $18(W Z)$.

Figura 3.13: PSNR do frame Wyner-Ziv para números diferentes de iterações do decodificador LIA, para sequência News, com QP = 25 (intra) e TQ = 18 (WZ), em uma escala maior (melhor visualização).

Figura 3.14: Diferença entre a PSNR do frame Wyner-Ziv utilizando uma e cinquenta iterações, para a sequência News, com QP = 25 (intra) e TQ = $18(W Z)$.

Figura 3.15: PSNR do frame Wyner-Ziv para números diferentes de iterações do decodificador LIA, para sequência Foreman, com QP = 26 (intra) e TQ = 7 (WZ).

Figura 3.16: Diferença entre a PSNR do frame Wyner-Ziv utilizando uma e cinquenta iterações, para a sequência Foreman, com QP = 26 (intra) $\mathrm{e} \mathrm{TQ}=7(\mathrm{WZ})$. 
Figura 3.17: PSNR do frame Wyner-Ziv para números diferentes de iterações do decodificador LIA, para sequência News, com QP = 26 (intra) $\mathrm{e} \mathrm{TQ}=7$ (WZ).

Figura 3.18: Diferença entre a PSNR do frame Wyner-Ziv utilizando uma e cinquenta iterações, para a sequência News, com QP = 26 (intra) $\mathrm{e} \mathrm{TQ}=7$ (WZ).

Figura 4.1: Diagrama de blocos da arquitetura de codificação distribuída proposta.

Figura 4.2: Ordem das posições (frequências espaciais) dentro de um bloco 4x4 de coeficientes DCT.

Figura 4.3: Quantizador escalar uniforme para o coeficiente DC com largura $W$ do intervalo de quantização.

Figura 4.4: Distribuição dos coeficientes DCT para a banda DC $\left(b_{1}\right)$ da sequência Foreman.

Figura 4.5: Distribuição dos coeficientes DCT para a banda DC $\left(b_{1}\right)$ da sequência News.

Figura 4.6: Distribuição dos coeficientes DCT para a banda DC $\left(b_{1}\right)$ da sequência Coastguard.

Figura 4.7: Distribuição dos coeficientes DCT para a banda DC $\left(b_{1}\right)$ da sequência Soccer.

Figura 4.8: Distribuição dos coeficientes DCT para a banda AC $\left(b_{2}\right)$ de mais baixa frequência espacial da sequência Foreman, QCIF.

Figura 4.9: Distribuição dos coeficientes DCT para a banda AC $\left(b_{2}\right)$ de mais baixa frequência espacial da sequência News, QCIF.

Figura 4.10: Distribuição dos coeficientes DCT para a banda AC $\left(b_{2}\right)$ de mais baixa frequência espacial da sequência Coastguard, QCIF.

Figura 4.11: Distribuição dos coeficientes DCT para a banda AC $\left(b_{2}\right)$ de mais baixa frequência espacial da sequência Soccer, QCIF.

Figura 4.12: Distribuição dos coeficientes DCT para a banda AC $\left(b_{3}\right)$ de mais alta frequência espacial da sequência Foreman QCIF.

Figura 4.13: Distribuição dos coeficientes DCT para a banda AC $\left(b_{3}\right)$ de mais alta frequência espacial da sequência News, QCIF.

Figura 4.14: Distribuição dos coeficientes DCT para a banda AC $\left(b_{3}\right)$ 
de mais alta frequência espacial da sequência Coastguard, QCIF.

Figura 4.15: Distribuição dos coeficientes DCT para a banda AC $\left(b_{3}\right)$

de mais alta frequência espacial da sequência Soccer, QCIF.

Figura 4.16: Quantizador escalar uniforme sem um intervalo de quantização simétrico em torno da amplitude zero.

Figura 4.17: problema de quantização dos coeficientes AC usando um quantizador sem um intervalo de quantização simétrico em torno da amplitude zero.

Figura 4.18: Quantizador escalar uniforme com um intervalo de quantização simétrico em torno da amplitude zero.

Figura 4.19: 18 matrizes de quantização associadas a diferentes desempenhos taxa-distorção, onde cada elemento da mesma é o número de níveis de quantização da banda correspondente àquela posição.

Figura 4.20: 8 matrizes de quantização associadas a diferentes desempenhos taxa-distorção, onde cada elemento da mesma é o número de níveis de quantização da banda correspondente àquela posição.

Figura 4.21: PSNR do frame Wyner-Ziv utilizando diferentes técnicas de quantização, para sequência Foreman, com QP = 26 (intra) e TQ = 18 (nível de qualidade).

Figura 4.22: PSNR do frame Wyner-Ziv utilizando diferentes técnicas de quantização, para sequência News, com QP = 26 (intra) e TQ = 18 (nível de qualidade).

Figura 4.23: PSNR do frame Wyner-Ziv utilizando diferentes técnicas de quantização, para sequência Coastguard, com QP = 26 (intra) e TQ = 18 (nível de qualidade).

Figura 4.24: PSNR do frame Wyner-Ziv utilizando diferentes técnicas de quantização, para sequência Soccer, com QP = 26 (intra) e TQ = 18 (nível de qualidade).

Figura 4.25: PSNR do frame Wyner-Ziv utilizando diferentes técnicas de quantização, para sequência Foreman, com QP = 36 (intra) e TQ $=7$ (nível de qualidade).

Figura 4.26: PSNR do frame Wyner-Ziv utilizando diferentes técnicas de quantização, para sequência News, com QP = 36 (intra) 
e TQ = 7 (nível de qualidade).

Figura 4.27: PSNR do frame Wyner-Ziv utilizando diferentes técnicas de quantização, para sequência Coastguard, com QP = 36 (intra) e TQ $=7$ (nível de qualidade).

Figura 4.28: PSNR do frame Wyner-Ziv utilizando diferentes técnicas de quantização, para sequência Soccer, com QP = 36 (intra) e TQ = 7 (nível de qualidade).

Figura 4.29: PSNR do frame Wyner-Ziv codificando-se diferentes quantidades de bitplanes, para sequência Foreman, com QP = 27 (intra) e TQ = 18 (nível de qualidade).

Figura 4.30: PSNR do frame Wyner-Ziv codificando-se diferentes quantidades de bitplanes, para sequência News, com QP = 27 (intra) e TQ = 18 (nível de qualidade).

Figura 4.31: (a) frame WZ original da sequência Foreman; (b)

frame WZ reconstruído considerando todos os bitplanes; (c) excluindo-se o bitplane menos significativo; (d) excluindo-se os dois bitplanes menos significativos; (e) excluindo-se os três bitplanes menos significativos.

Figura 4.32: (a) frame WZ original da sequência News; (b) frame WZ reconstruído considerando todos os bitplanes; (c) excluindo-se o bitplane menos significativo; (d) excluindo-se os dois bitplanes menos significativos; (e) excluindo-se os três bitplanes menos significativos.

Figura 4.33: Probabilidade condicional do bit $x_{2, j}$ dada a informação lateral $y_{j}$ e que o bit anterior, $x_{1, j}$, foi estimado com valor igual 1 .

Figura 4.34: PSNR para diferentes níveis de estimação do parâmetro laplaciano, para a sequência Foreman, QCIF, com QP = 32 (intra) e TQ $=9$ (WZ).

Figura 4.35: PSNR para diferentes níveis de estimação do parâmetro laplaciano, para a sequência News, QCIF, QP = 32 (intra) e TQ = 9 (WZ). 136 Figura 4.36: PSNR para diferentes níveis de estimação do parâmetro laplaciano, sequência Coastguard, QCIF, QP = 32 (intra) e TQ = 9 (WZ).

Figura 4.37: PSNR para diferentes níveis de estimação do parâmetro laplaciano, para a sequência Soccer, QCIF, QP = 32 (intra) e TQ = 9 (WZ). 
Figura 4.38: PSNR para decodificação com e sem a utilização da modelagem do canal de correlação, para a sequência Foreman, QCIF, com $\mathrm{QP}=32$ (intra) e TQ = 9 (WZ).

Figura 4.39: PSNR para decodificação com e sem a utilização da modelagem do canal de correlação, para a sequência News, QCIF, com QP = 32 (intra) e TQ = 9 (WZ).

Figura 4.40: hipóteses de estimação de movimento do frame atual.

Figura 4.41: Processo de geração da informação lateral Proposto

Figura 4.42: Componentes básicos do processo de estimação direta de movimento

Figura 4.43: (a) estimação direta do movimento passando pelo frame interpolado $\mathrm{Y}_{2 \mathrm{i}}$. (b) projeção dos blocos utilizados na estimação do movimento.

Figura 4.44: interpolação a partir do processo de compensação do movimento, apresentando várias estimações associadas a um único ponto do frame interpolado.

Figura 4.45: Sentido e direção dos vetores de movimento na estimação reversa.

Figura 4.46: PSNR da informação lateral ao longo da sequência para diferentes métodos de estimação de movimento, sequência Foreman, com QP = 40 (intra), macrobloco 8x8.

Figura 4.47: PSNR da informação lateral ao longo da sequência para diferentes métodos de estimação de movimento, sequência News, com QP = 40 (intra), macrobloco 8x8.

Figura 4.48: Número médio de buscas por macrobloco, para diferentes métodos, para a sequência Foreman, com QP = 40 (intra), macrobloco 8x8.

Figura 4.49: Número médio de buscas por macrobloco, para diferentes métodos, para a sequência News, com QP = 40 (intra), macrobloco 8x8.

Figura 4.50: PSNR da informação lateral ao longo da sequência para diferentes métodos de estimação de movimento, sequência Foreman, com QP = 26 (intra), macrobloco 8x8.

Figura 4.51: PSNR da informação lateral ao longo da sequência para diferentes métodos de estimação de movimento, sequência News, com 
$\mathrm{QP}=26$ (intra), macrobloco 8x8.

Figura 4.52: Número médio de buscas por macrobloco, para diferentes métodos, para a sequência Foreman, com QP = 26 (intra), macrobloco 8x8. 154 Figura 4.53: Número médio de buscas por macrobloco, para diferentes métodos, para a sequência News, com QP = 26 (intra), macrobloco 8x8.

Figura 4.54: PSNR da informação lateral para diferentes métodos de estimação de movimento, sequência Foreman, com QP = 40 (intra), macrobloco $16 \times 16$.

Figura 4.55: PSNR da informação lateral ao longo da sequência para diferentes métodos de estimação de movimento, sequência News, com QP = 40 (intra), macrobloco 16x16.

Figura 4.56: Número médio de buscas por macrobloco, para diferentes métodos, sequência Foreman, com QP = 40 (intra), macrobloco 16x16.

Figura 4.57: Número médio de buscas por macrobloco, para diferentes métodos, para a sequência News, com QP = 40 (intra), macrobloco 16x16.

Figura 4.58: PSNR da informação lateral para diferentes métodos de estimação de movimento, sequência Foreman, com QP = 26 (intra), macrobloco $16 \times 16$.

Figura 4.59: PSNR da informação lateral ao longo da sequência para diferentes métodos de estimação de movimento, sequência News, com $\mathrm{QP}=26$ (intra), macrobloco 16x16.

Figura 4.60: Número médio de buscas por macrobloco, para diferentes métodos, sequência Foreman, com QP = 26 (intra), macrobloco 16x16.

Figura 4.61: Número médio de buscas por macrobloco, para diferentes métodos, para a sequência News, com QP = 26 (intra), macrobloco 16x16.

Figura 4.62: Pixels descobertos no frame interpolado devido à estimação de movimento não partir do centro dos blocos do frame interpolado [70].

Figura 4.63: Exemplo de disposição de blocos resultantes da estimação e compensação do movimento no frame interpolado.

Figura 4.64: Disposição dos blocos resultantes da compensação de movimento, no frame interpolado: (a) utilizando blocos 8x8; (b) utilizando blocos 16x16.

Figura 4.65: Método de extrapolação proposto neste trabalho. 
Figura 4.66: Frame WZ reconstruído utilizando a seguinte técnica de geração da informação lateral: (a) Interpolação simples;

(b) Interpolação ponderada (proposta); (c) Método proposto

(d) Frame WZ original da sequência Foreman.

Figura 4.67: Frame WZ 138 reconstruído utilizando a seguinte técnica de geração da informação lateral: (a) interpolação simples;

(b) Interpolação ponderada (proposta); (c) Método proposto

(d) Frame WZ original da sequência News.

Figura 4.68: Frame WZ 138 reconstruído utilizando a seguinte técnica de geração da informação lateral: (a) interpolação simples;

(b) Interpolação ponderada (proposta); (c) Método proposto

(d) Frame WZ original da sequência Soccer.

Figura 4.69: Frame WZ 138 reconstruído utilizando a seguinte técnica de geração da informação lateral: (a) interpolação simples;

(b) Interpolação ponderada (proposta); (c) Método proposto

(d) Frame WZ original da sequência Coastguard.

Figura 4.70: PSNR para as diversas etapas do processo de geração da informação lateral, para a sequência Foreman, QCIF, com passo de quantização QP = 24 (intra).

Figura 4.71: PSNR para as diversas etapas do processo de geração da informação lateral, para a sequência News, QCIF, com passo de quantização QP = 24 (intra).

Figura 4.72: PSNR para as diversas etapas do processo de geração da informação lateral, para a sequência Coastguard, QCIF, com passo de quantização QP = 24 (intra).

Figura 4.73: PSNR para as diversas etapas do processo de geração da informação lateral, para a sequência Soccer, QCIF, com passo de quantização QP = 24 (intra).

Figura 4.74: PSNR para as diversas etapas do processo de geração da informação lateral, para a sequência Foreman, QCIF, com passo de quantização QP = 38 (intra).

Figura 4.75: PSNR para as diversas etapas do processo de geração da informação lateral, para a sequência News, QCIF, com passo de 
quantização QP = 38 (intra).

Figura 4.76: PSNR para as diversas etapas do processo de geração da informação lateral, para a sequência Coastguard, QCIF, com passo de quantização QP = 38 (intra).

Figura 4.77: PSNR para as diversas etapas do processo de geração da informação lateral, para a sequência Soccer, QCIF, com passo de quantização QP = 38 (intra).

Figura 4.78: PSNR do frame Wyner-Ziv utilizando diferentes fatores de normalização, para sequência Foreman, com QP = 38 (intra) $\mathrm{e} \mathrm{TQ}=7$ (WZ).

Figura 4.79: PSNR do frame Wyner-Ziv utilizando diferentes fatores de normalização, para sequência News, com QP = 38 (intra) $\mathrm{e} \mathrm{TQ}=7$ (WZ).

Figura 4.80: PSNR do frame Wyner-Ziv utilizando diferentes fatores de normalização, para sequência Coastguard, com QP = 38 (intra) $\mathrm{e} \mathrm{TQ}=7$ (WZ).

Figura 4.81: PSNR do frame Wyner-Ziv utilizando diferentes fatores de normalização, para sequência Soccer, com QP = 38 (intra) $\mathrm{e} \mathrm{TQ}=7$ (WZ).

Figura 4.82: Comparação entre o valor médio dos pixels do frame WZ e da informação lateral, para sequência News.

Figura 4.83: Comparação entre o valor médio dos pixels do frame WZ e da informação lateral, para sequência Foreman.

Figura 4.84: Frames resultantes do processo de: (a) interpolação ponderada da estimação bidirecional de movimento 8x8;

(b) interpolação ponderada da estimação bidirecional de movimento 16x16; (c) extrapolação do frame interpolado; (d) normalização da informação lateral; (e) frame WZ original.

Figura 4.85: Procedimento de reconstrução de cada coeficiente DCT da banda $b_{k}$ : (a) Caso I, (b) Caso II, (c) Caso III.

Figura 4.86: PSNR média para a sequência Foreman, para 3 níveis diferentes de qualidade (QP, TQ) versus tempo de processamento.

Figura 4.87: Variação da PSNR do frames WZ, ao longo dos da 
sequência Foreman, para diferentes passos de quantização intraframe e diferentes qualidades: $\{\mathrm{QP}=24, \mathrm{TQ}=18\},\{\mathrm{QP}=28, \mathrm{TQ}=10\},\{\mathrm{QP}=32, \mathrm{TQ}=4\} .185$ Figura 4.88: PSNR do frame Wyner-Ziv, substituindo-se as bandas com número de níveis igual a zero pelas correspondentes da informação lateral, para sequência News, com QP = 28 (intra) e TQ = 13 (WZ). 186

Figura 4.89: PSNR do frame Wyner-Ziv, substituindo-se as bandas

com número de níveis igual a zero pelas correspondentes da informação lateral, para sequência News, com QP = 28 (intra) e TQ = 13 (WZ). 


\section{Lista de Tabelas}

Tabela 4.1: Métodos de cálculo da faixa dinâmica do coeficiente DC

105

Tabela 4.2: Composição dos bitplanes da k-ésima banda.

Tabela 4.3: número total de bits transmitidos por banda, conforme o número de bitplanes codificados.

Tabela 4.4: PSNR média e número de parâmetros laplacianos que necessitam ser calculados em função do nível de granularidade. 


\section{Lista de Algoritmos}

Algoritmo 4.1: cálculo do passo de quantização baseado no método dvc

106

Algoritmo 4.2: cálculo do passo de quantização para o método proposto

Algoritmo 4.3: verificação do grau de confiabilidade em relação aos bits decodificados

Algoritmo 4.4: cálculo do frame interpolado

145

Algoritmo 4.5: interpolação baseada na quantidade de movimento

161

Algoritmo 4.6: extrapolação de pixels

162

Algoritmo 4.7: reconstrução dos coeficientes DCT 


\section{Lista de Siglas e Abreviaturas}

\begin{tabular}{|c|c|}
\hline ADSL & Asynchronous Digital Subscriber Line \\
\hline APP & A Posteriori Probability \\
\hline ARPS & Adaptive Rood Pattern Search \\
\hline AVC & Advanced Video Coding \\
\hline BCJR & Bahl-Cocke-Jelinek-Raviv \\
\hline BP & Belief Propagation \\
\hline Codec & Codificador e decodificador \\
\hline Coset & Codeword set \\
\hline CRC & Cyclic Redundancy Check \\
\hline DCT & Discrete Cosine Transform \\
\hline DS & Diamond Search \\
\hline DSC & Distributed Source Coding \\
\hline DVC & Distributed Video Coding \\
\hline eIRA & extended Irregular Repeat Accumulate \\
\hline ES & Exhaustive Search \\
\hline GOP & Group of Pictures \\
\hline HVS & Human Visual System \\
\hline IDCT & Inverse Discrete Cosine Transform \\
\hline ITU-T & International Telecommunication Union - Telecomunications \\
\hline KLT & Transformada de Karhunen-Loève \\
\hline LDPC & Low Density Parity-Check \\
\hline LDPCA & LDPC Acumulado \\
\hline LIA & LDPC Irregular, Acumulado e Adaptativo \\
\hline LLR & Log-Likelihood Ratio \\
\hline LSB & Least Significant Bits \\
\hline MAD & Mean Absolute Difference \\
\hline MAP & Maximum A Posteriori \\
\hline ML & Maximum Likelihood \\
\hline MMS & Multimedia Messaging Service \\
\hline MPEG & Moving Picture Experts Group \\
\hline
\end{tabular}


MSB Most Significant Bits

MSE Mean Square Error

MV Motion Vector

NCC Número de Casos Confiáveis

NTSS New Three Step Search

PDWZ Pixel Domain Wyner-Ziv

PRISM Power-efficient, Robust, high compression, Syndrome-based

Multimedia (coding)

PSNR Peak Signal-to-Noise Ratio

QCIF Quarter Common International Format

QP Quantization Parameter

RCPT Rate-Compatible Punctured Turbo

RD Rate-Distortion

SISO Soft Input-Soft Output

SP Sum-Product

SES Simple and Efficient TSS

4SS Four Step Search

TDWZ Transform Domain Wyner-Ziv

TQ Tabela de Quantização

TSS Three Step Search

WZ Wyner-Ziv 